\title{
Article
}

\section{Improving hand vein recognition by score weighted fusion of wavelet-domain multi- radius local binary patterns}

\author{
Wang, Y, Duan, Q, Shark, L and Huang, D \\ Available at http://clok.uclan.ac.uk/15739/ \\ Wang, Y, Duan, Q, Shark, L ORCID: 0000-0002-9156-2003 and Huang, D \\ (2016) Improving hand vein recognition by score weighted fusion of wavelet- \\ domain multi-radius local binary patterns. International Journal of Computer \\ Applications in Technology, 54 (2). pp. 151-160. ISSN 0975-8887
}

It is advisable to refer to the publisher's version if you intend to cite from the work.

For more information about UCLan's research in this area go to http://www.uclan.ac.uk/researchgroups/ and search for < name of research Group>.

For information about Research generally at UCLan please go to http://www.uclan.ac.uk/research/

All outputs in CLoK are protected by Intellectual Property Rights law, including Copyright law. Copyright, IPR and Moral Rights for the works on this site are retained by the individual authors and/or other copyright owners. Terms and conditions for use of this material are defined in the policies page.

\section{CLoK}

Central Lancashire online Knowledge www.clok.uclan.ac.uk

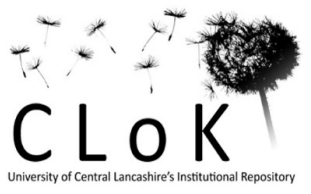




\title{
Improving hand vein recognition by score weighted fusion of wavelet-domain multi-radius local binary patterns
}

\section{Yiding Wang and Qiangyu Duan}

School of Electronic and Information Engineering,

North China University of Technology,

Beijing 100144, China

Email: wangyd@ncut.edu.cn

Email: dqy1014@sina.cn

\section{Lik-Kwan Shark*}

Applied Digital Signal \& Image Processing Research Centre,

School of Engineering,

University of Central Lancashire,

Preston PR1 2HE, UK

Email: 1shark@uclan.ac.uk

*Corresponding author

\section{Di Huang}

School of Computer Science and Engineering,

Beihang University,

Beijing 100191, China

Email: dhuang@buaa.edu.cn

\begin{abstract}
Among biometric modalities, hand vein patterns are seen as providing an attractive method for high-level security access applications owing to high impenetrability and good user convenience. For biometric recognition based on near-infrared dorsal hand vein images, Local Binary Patterns (LBP) have emerged as a highly effective descriptor of local image texture with high recognition performance reported. In this paper, the traditional approach with LBP applied in the spatial domain is extended to multi-radius LBP in the wavelet domain to provide a more comprehensive set of feature categories to capture grey-level variation characteristics of vein patterns, and score weighted fusion based on the relative discriminative power of each feature category is proposed to achieve higher recognition performance. The proposed methodology is shown to provide a more robust performance with a recognition rate in excess of $99 \%$ and an equal error rate significantly less than $2 \%$.
\end{abstract}

Keywords: biometrics; local binary patterns; score weighted fusion; hand vein recognition; wavelet transform.

Reference to this paper should be made as follows: Wang, Y., Duan, Q., Shark, L-K. and Huang, D. (2016) 'Improving hand vein recognition by score weighted fusion of wavelet-domain multi-radius local binary patterns', Int. J. Computer Applications in Technology, Vol. 54, No. 3, pp.151-160.

Biographical notes: Yiding Wang is a Professor at the School of Electronic and Information Engineering, North China University of Technology, Beijing, China. He received his PhD from Nanjing University of Science and Technology, China, in 1998. His main research areas include intelligent recognition and image processing, radio location technology and industrial nondestructive testing.

Qiangyu Duan obtained his Bachelor's degree from Xinxiang Medical University, China, in 2009 and he is studying for his Master's degree in North China University of Technology. His main research areas include digital image processing and pattern recognition.

Lik-Kwan Shark received his BSc (Hons) in Electronic and Communications Engineering from the Polytechnic of North London in 1983, his MSc in Microwave and Modern Optics from University College London in 1984 and his PhD in Digital Satellite Systems from Lancashire Polytechnic in collaboration with the Royal Aircraft Establishment at Farnborough in 1988. He 
has been the Chair Professor of Signal and Image Processing at the University of Central Lancashire since 2001, and heads two research centres, which are the Applied Digital Signal and Image Processing Research Centre (ADSIP) and the Advanced Digital Manufacturing Technology Research Centre (ADMT).

Di Huang received the BS and MS degrees in Computer Science from Beihang University, Beijing, China, and the $\mathrm{PhD}$ degree in Computer Science from Ecole centrale de Lyon, Lyon, France, in 2005, 2008, and 2011, respectively. He then joined the Laboratory of Intelligent Recognition and Image Processing with the Beijing Key Laboratory of Digital Media, School of Computer Science and Engineering, Beihang University, as a faculty member. He is currently Associate Professor and his research interests include biometrics, in particular, on 2D/3D face analysis, image/video processing, and pattern recognition.

\section{Introduction}

Among biometric modalities which have been increasingly used to replace traditional personal identification methods based on keys, cards and passwords, hand vein patterns are seen as providing an attractive method for high-level security access applications (Delac and Grgic, 2004; Ding et al., 2005; Shah et al., 2015; Wang et al., 2007; Wang et al., 2015). In addition to offering the same convenience (without the need of carrying keys or cards and memorising passwords) and same security (with higher impenetrability against stealing or falsification) like other biometric modalities (Lin and Fan, 2004), hand vein based biometrics possess a number of unique advantages. In particular, hand veins are much harder to forge through liveness detection (Wang and Zhao, 2013); are less susceptible to surface conditions affected by dirt, scratches and wear; and are less intrusive owing to contactless imaging.

For biometric recognition using Near-Infrared (NIR) dorsal hand vein images, the approaches found in the literature use predominantly geometry-based features extracted from vein patterns. With vein patterns represented by their medial axes and employing constrained sequential correlation, Cross and Smith (1995) achieved a False Rejection Rate (FRR) of $7.5 \%$ and a False Acceptance Rate (FAR) of $0 \%$ based on 100 images from 20 subjects. With vein patterns represented by their minutiae points and employing the Hausdorff distance, Wang et al. (2008) showed the possibility of achieving an FRR and an FAR of $0 \%$ for 47 subjects. Based on the topological structure from the hand vein minutiae extracted using Delaunay triangulation and the geometrical features obtained from knuckle tips, Kumar and Prathyusha (2009) achieved an Equal Error Rate (EER) of $1.14 \%$ based on 300 images from 100 subjects. Recently, based on the key points extracted from vein patterns using the scale-invariant feature transform, an EER of $0.81 \%$ has been reported based on 2040 images from 204 hands (Wang et al., 2014). The biometric recognition performances of these methods are largely limited by the geometrical visibility of vein patterns. Since not all the dorsal hand veins can be made clearly visible without being affected by illumination conditions and hand postures, the vein patterns extracted from dorsal hand vein images are prone to error. Some thin veins could be missed, and some extra veins could be generated in dark image regions. Compounded by the additional factor of a limited number of vein lines visible under
NIR (often much less than 10 per hand), small errors could become significant with a considerable impact on the recognition performance.

An alternative approach is to use the grey-level appearance based on global or local measures. Applying independent component analysis to the global grey-level appearance of dorsal hand vein images has been shown to provide a better recognition performance than those approaches employing geometry-based features (Yuksel et al., 2011). Among the various approaches based on local grey-level appearance (Ojala et al., 1996), Local Binary Patterns (LBP) (Ojala et al., 2002) have emerged as a highly effective descriptor of local image texture in dorsal hand vein images, achieving a recognition performance around 98\% (Wang et al., 2010; Wang et al., 2011). This has led to the work described in this paper to extend the LPB approach to achieve a higher level of recognition performance for NIR dorsal hand vein images.

From the perspective of image feature representation for hand vein based personal identification, a weakness of LBP is in its small coverage area of local grey-level variation characteristics. This has led to the work in this paper to extend LBP computed at a fixed sampling radius in the image domain in the traditional approach to the proposed approach with LBP computed at multiple sampling radii in the transformed wavelet domain, thereby providing additional texture categories at different orientations and scales with a larger coverage area to the hand vein classification stage. Since different texture categories and different coverage areas capture different aspects of grey-level variation characteristics of hand vein patterns and result in different classification performance, also proposed in this paper is score weighted fusion to yield an overall recognition performance that is higher than those based on individual texture categories. Although wavelet-based LBP has been previously proposed (Song and Li, 2013; Mu et al., 2011), this paper extends it to multi-radius wavelet LBP for image classification based on discriminative power of LBP computed at individual sampling radius in both spatial and wavelet domains. Furthermore, wavelet LBP has not been investigated for biometric recognition based on hand vein patterns.

The rest of this paper is organised as follows. Section 2 presents the dorsal hand vein images as well as necessary geometric and grey-level pre-processing operations required before feature extraction and recognition, and Section 3 presents the proposed recognition methodology based on 
wavelet and LBP plus score weighted fusion. These are then followed by Section 4 with experimental results to demonstrate the recognition performance of the proposed approach. Finally, the paper ends with the conclusions in Section 5.

\section{Dorsal hand vein images and pre-processing}

In this work, the dorsal hand vein images were acquired using the NIR imaging device developed by the authors (Wang et al., 2014). During biometric acquisition, each user is required to grasp a handle under a box, which contains Light Emitting Diodes (LED) to provide the NIR illumination at a wavelength of $850 \mathrm{~nm}$, and a camera to capture the NIR light bounced off the back of the hand. A database was established by collecting dorsal hand vein images from 102 volunteers. In order to capture intra-class variation, each volunteer was required to grasp the handle 20 times by using their left hand and right hand alternately during image capture. This results in ten images acquired from each hand with a total of 2040 dorsal hand vein images in the database. The images are stored with a resolution of $640 \times 480$ pixels, and a sample image of the database is shown in Figure 1(a). The database is publicly available and has been used by researchers working on vein biometrics (Honarpisheh and Faez, 2013; Zhu and Huang, 2012; Tang et al., 2012; Zhu et al., 2013).

Figure 1 (a) sample image; (b) shearing computation; (c) shear corrected image with centroid and extracted ROI shown in square; and (d) filtered ROI

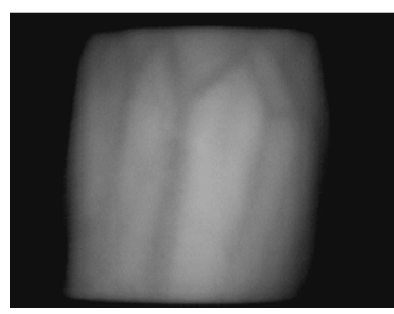

(a)

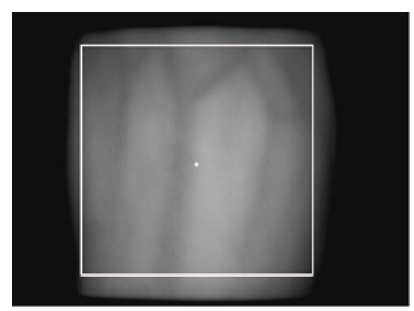

(c)

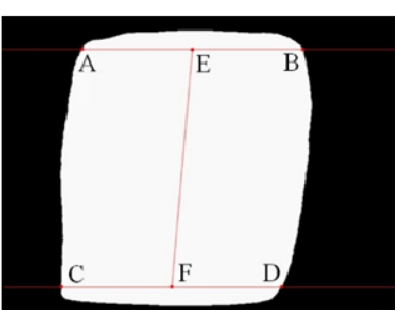

(b)

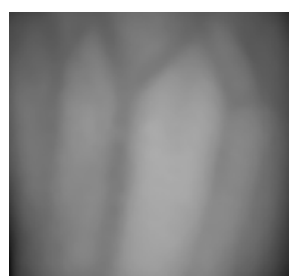

(d)
There are two stages in pre-processing of dorsal hand vein images, which are geometric processing followed by greylevel processing. The aim of geometric processing is to extract the same part of the hand-dorsal region from the images acquired, such that biometric recognition is less affected by geometric variations of vein patterns owing to inconsistent hand positions and postures with respect to the camera. Although the handle for grasping during vein image capture has restricted the hand position and posture, a slight horizontal shearing effect, as shown in Figure 1(a), has been found to occur in some of the acquired dorsal hand vein images owing to a small lateral wrist movement. This horizontal shear can be corrected by using

$$
\left[\begin{array}{ll}
x & y
\end{array}\right]=\left[\begin{array}{ll}
x^{\prime} & y^{\prime}
\end{array}\right]\left[\begin{array}{ll}
1 & 0 \\
t & 1
\end{array}\right]
$$

where $\left(x^{\prime}, y^{\prime}\right)$ denotes the pixel coordinates in the horizontally sheared image (acquired image), $(x, y)$ the pixel coordinates after geometric image correction and $t$ the shearing factor.

In order to determine the shearing factor, the Ostu thresholding method (Otsu, 1979) is applied to the acquired image to yield a threshold, and the threshold is then used to convert the acquired image into a binary image with 1 corresponding to the hand-dorsal region and 0 corresponding to the background as shown in Figure 1(b). Subsequently, four boundary points along two pre-defined horizontal lines near the top and bottom of the binary image are found based on the first transition from black to white and the last transition from white to black along the two horizontal lines. This is illustrated in Figure 1(b) with points $A, B, C$ and $D$ denoting the four boundary points found. Based on the imaging distance between the camera and hand, the horizontal lines were empirically fixed at 30 lines below the top image border and above the bottom image border. Using the middle points of $A B$ and $C D$ calculated and denoted as $E$ and $F$, the shearing factor $t$ is estimated as the slope of line $E F$.

After the horizontal shear correction using equation (1), the image centroid $\left(x_{o}, y_{o}\right)$ is used as the geometric reference for extraction of a consistent Region of Interest (ROI) and computed using

$$
x_{o}=\frac{\sum_{x} \sum_{y} x \cdot I(x, y)}{\sum_{x} \sum_{y} I(x, y)} ; \quad y_{o}=\frac{\sum_{x} \sum_{y} y \cdot I(x, y)}{\sum_{x} \sum_{y} I(x, y)}
$$

where $I(x, y)$ denotes the pixel value at position $(x, y)$ of the shear corrected image. As an example, Figure 1(c) shows the corresponding image with geometric correction and the ROI extracted from the acquired image shown in Figure 1(a). Finally, using the nearest neighbour interpolation, the size of the extracted ROI is normalised to $256 \times 256$ pixels.

For grey-level processing, there are two objectives: the first is to increase image contrast and the second is to minimise image noise, so as to enable extraction of characteristic greylevel variation of vein patterns. For the first objective, a rectangular pulse with the pulse width matched to the average vein width is used as the matched filter and applied to each row of the dorsal hand vein image to maximise the peak response upon encountering a vein. For the second objective, a 2D Wiener filter is used to minimise noise based on a local window with the window size set to the average vein width. Based on the average vein width of 15 pixels, Figure 1(d) shows the filtered image of the extracted ROI shown in Figure 1(c) as an example, where the filtered image is seen to have a higher contrast between veins and background tissue, as well as more uniform greylevel appearance of vein lines and background tissue. 


\section{Recognition method}

The proposed methodology for biometric recognition of dorsal hand vein images consists of wavelet transform to yield a multi-scale representation of image features at different orientations, multi-radius LBP to yield a feature vector for each of the informative wavelet decomposed image, and score weighted fusion to yield a high recognition performance.

\subsection{Wavelet transform of hand vein images}

Wavelet transform of an image provides a hierarchical set of sub-band images containing the feature information captured at different orientations and resolutions. Based on the separability property of the wavelet transform as a result of using orthogonal basis functions, the discrete wavelet transform of an image can be implemented as two 1D wavelet transforms, by using a two-channel filter bank consisting of a lowpass filter and a highpass filter with their impulse responses derived from a particular wavelet basis function, as shown in Figure 2. The first 1D wavelet transform is performed along the horizontal direction of each image with each row being treated as a 1D signal. By performing lowpass and highpass operations on each row followed by a 2-to-1 down-sampling operation to discard every other column, the first 1D wavelet transform produces two outputs for each image, namely the horizontal lowpass output and the horizontal highpass output. The second 1D wavelet transform is performed along the vertical direction of the outputs produced by the first 1D wavelet transform with each column of the horizontal lowpass output and the horizontal highpass output being treated as a 1D signal. By performing lowpass and highpass operations on each column followed by a 2-to-1 down-sampling operation to discard every other row, the second 1D wavelet transform produces four first-level wavelet components for each image, namely the vertical lowpass of the horizontal lowpass output denoted by $w_{1, a}$, the vertical highpass of the horizontal lowpass output denoted by $w_{1, h}$, the vertical lowpass of the horizontal highpass output denoted by $w_{1, v}$ and the vertical highpass of the horizontal highpass output denoted by $w_{1, d}$. While $w_{1, a}$ corresponds to the first approximation of the input image, $w_{1, h}, w_{1, v}$ and $w_{1, d}$ correspond to the horizontal, diagonal and vertical detail components, respectively. Applying the whole process again to $w_{1, a}$ yields four further wavelet components of $w_{2, o}$ at the second image decomposition level with $o \in[a, h, v, d]$ denoting the orientation, and the entire process can be repeated until the desired image decomposition level is reached.

Figure 2 Wavelet based image decomposition

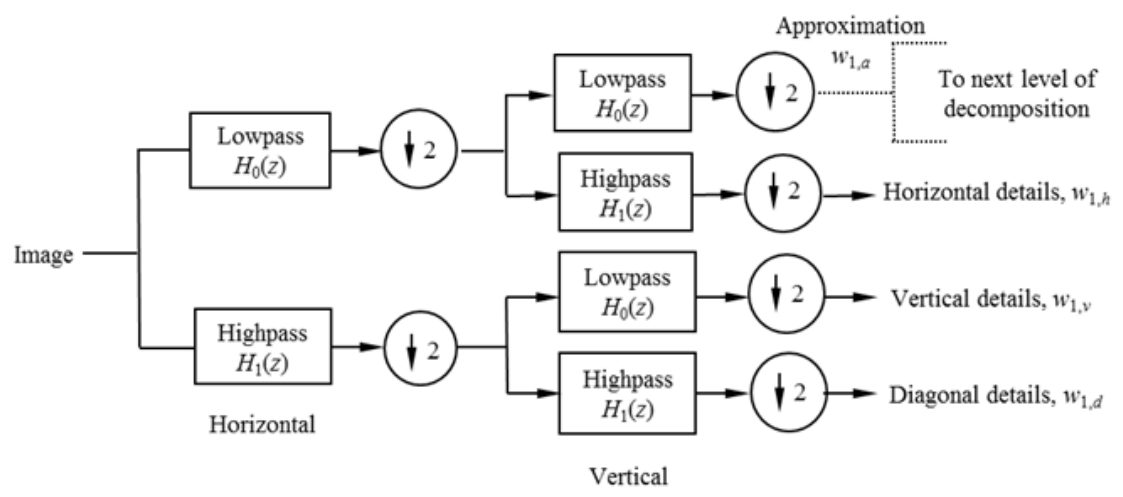

Figure 3 Wavelet decomposed images

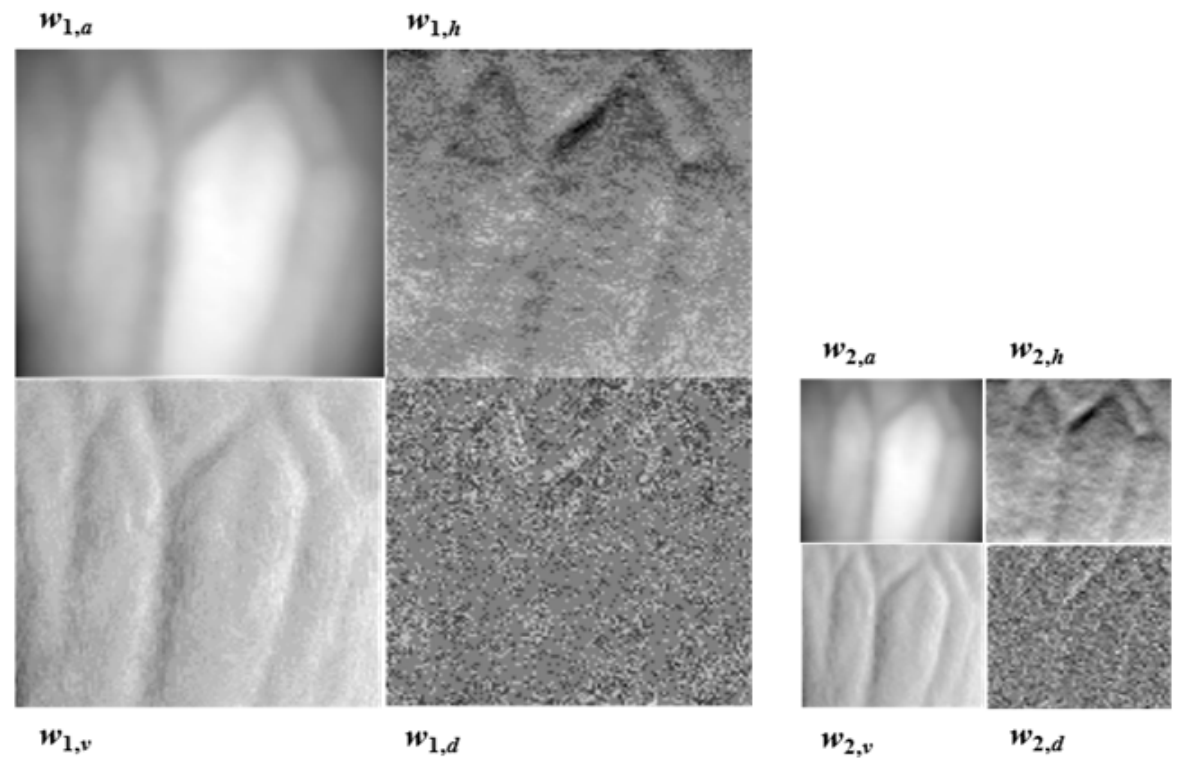


In implementing the discrete wavelet transform of dorsal hand vein images, the Haar wavelet was selected, since its rectangular basis function offers an advantage for localisation of vein edges in images and it is also the simplest wavelet for computation compared with other wavelets. Furthermore, the number of decomposition levels was set to two without increasing unnecessarily the number of additional feature categories. Using the extracted ROI shown in Figure 1(d) as an example, Figure 3 shows the output wavelet components produced by applying the two-level Haar wavelet transform. It is seen from Figure 3 that all the structure information of the vein pattern are contained in the approximation wavelet components at two different scales. Compared with the horizontal wavelet components, the vertical wavelet components are seen to contain more useful information of the vein structure, and this reflects the dominant orientation of most vein lines in the vertical direction. The diagonal wavelet components are seen to be least useful with very little vein structure visible.

\subsection{Multi-radius local binary patterns}

Proposed by Ojala et al. (2002), Local Binary Patterns (LBP) provide an efficient descriptor of local grey-level appearance. By treating a given pixel as the centre with its neighbourhood pixels defined as equally spaced sampling points on a circle from the centre pixel, the LBP generates a binary representation of the local grey-level appearance by comparing the centre pixel grey level with those of its neighbour pixels. This operation can be expressed mathematically as

$$
L B P_{P, R}=\sum_{p=0}^{P-1} s\left(g_{p}-g_{c}\right) 2^{p}
$$

with

$$
s\left(g_{p}-g_{c}\right)= \begin{cases}0 & \text { if } g_{p}-g_{c}<0 \\ 1 & \text { if } g_{p}-g_{c} \geq 0\end{cases}
$$

where $R$ denotes the radius of the circle from the centre pixel, $P$ the number of equally spaced sampling points on the circle, $g_{c}$ the grey level of the centre pixel and $g_{p}$ the grey level of each sampling point on the circle. If a sampling point does not fall exactly at the integer pixel positions, then $g_{p}$ is obtained by bilinear interpolation of its four nearest neighbouring pixels.

Although there are $2^{P}$ possible binary patterns which could be produced by the LBP operation, only a small subset of them is useful to describe the primitive grey-level variation patterns. One subset is based on the uniformity measure of LBP defined as the number of binary transitions from 0 to 1 or 1 to 0 between successive bits in the circular representation of the binary code, and is given by

$$
\begin{aligned}
U\left(L B P_{P, R}\right) & =\left|s\left(g_{P-1}-g_{c}\right)-s\left(g_{0}-g_{c}\right)\right| \\
& +\sum_{p=1}^{P+1}\left|s\left(g_{p}-g_{c}\right)-s\left(g_{p-1}-g_{c}\right)\right|
\end{aligned}
$$

With $U\left(L B P_{P, R}\right) \leq 2$, the subset of LBP is known as the uniform LBP which contains at most two binary transitions in its circular binary patterns as a result of two significant spatial grey-level changes, and it has $P(P-1)+3$ possible binary patterns.
Among the uniform LBP, there exist $P-1$ rotated versions of each binary pattern with the smallest binary value, owing to $P$ possible angular positions around the sampling circle. By removing the rotated versions, the number of possible binary patterns is reduced further to $P$, and the resulting rotation invariant uniform LBP is given by

$$
L B P_{P, R}^{r i U 2}= \begin{cases}\sum_{p=0}^{P-1} s\left(g_{p}-g_{c}\right) & \text { if } U\left(L B P_{P, R}\right) \leq 2 \\ P+1 & \text { otherwise }\end{cases}
$$

With $P=8$ and $R=2$, Figure 4 shows ten possible LBP categories indicated by their corresponding pattern numbers based on equation (6), where black and white circles indicate binary values of 0 and 1 . From Figure 4 , patterns 0 and 8 can be seen to indicate spot/flat area, patterns 1, 2, 6 and 7 indicate line ending points, patterns 3 and 5 indicate corner points, pattern 4 indicates edges and pattern 9 indicates all other possible non-uniform binary patterns (with grey circles indicating either binary 0 or 1 ).

By obtaining LBP for each pixel at position $(x, y)$ of an image of $N \times M$ pixels at radius $R$ in either spatial or wavelet domain, a normalised histogram indicating the percentage of each binary pattern can be constructed as the feature vector of the image, and is given by

$$
h_{k}=\frac{\sum_{x=1}^{N} \sum_{y=1}^{M} \delta\left[L B P_{P, R}^{r i U 2}(x, y)-k\right]}{N M}
$$

where $k \in[0, P+1]$. To capture the grey-level variation patterns within a larger area, multiple LBP histograms at different sampling radii are computed, and concatenated to form a joint histogram of the multi-radius LBP as the feature vector of the image. For example, for the multiradius LBP computed at sampling radii of 2, 3 and 5 pixels, their joint histogram is given by $H=\left[h_{k}^{2}, h_{k}^{3}, h_{k}^{5}\right]$.

An even more comprehensive feature vector can be provided by partition of each image in the spatial and wavelet domains into non-overlapping regions, and concatenation of the multi-radius LBP histograms from each region to provide position-based local grey-level appearance (Wang et al., 2010; Wang et al., 2011). In the implementation, the same image size is maintained for each partitioned block at different image scales. Hence, if the original image is divided into $L \times L$ nonoverlapping blocks, then each of the wavelet components is divided into $(L / 2) \times(L / 2)$ non-overlapping blocks at the first decomposition level, and $(L / 4) \times(L / 4)$ non-overlapping blocks at the second decomposition level. Ignoring the diagonal wavelet components as they have been found to be noninformative, concatenation of the resulting LBP histograms from each image produces seven different feature categories, namely $F_{0}(l, H)$ for the original image with $l \in[0, L \times L]$ denoting the index of the partitioned image blocks, $F_{1, o}(l, H)$ with $l \in[0,(L / 2) \times(L / 2)]$ and $o \in[a, h, v]$ denoting the orientation of the three wavelet components at the first decomposition level and $F_{2, o}(l, H)$ with $l \in[0,(L / 4) \times(L / 4)]$ for the three wavelet components at the second decomposition level. 
Figure 4 Local binary patterns from $L B P_{8,2}^{r i U 2}$

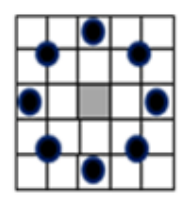

$L B P_{8,2}^{r i 2}=0$

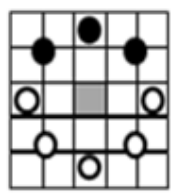

$L B P_{8,2}^{r i U^{2}}=5$

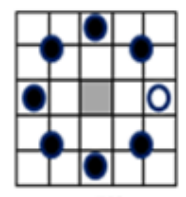

$L B P_{8,2}^{n i 2}=1$

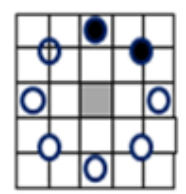

$L B P_{8,2}^{r i 2}=6$

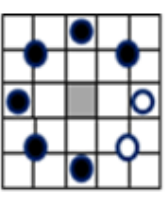

$L B P_{8,2}^{r i U 2}=2$

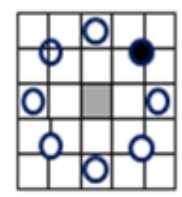

$L B P_{8,2}^{r i U 2}=7$

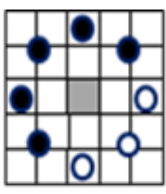

$L B P_{8,2}^{r i U 2}=3$

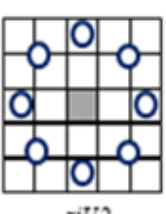

$L B P_{8,2}^{r i U 2}=8$

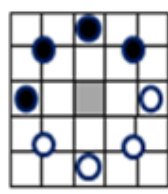

$L B P_{8,2}^{r i U / 2}=4$

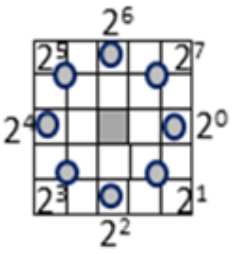

$L B P_{8,2}^{r i U 2}=9$

(non-uniform

patterns)

\subsection{Score weighted fusion}

As a result of applying multi-radius LBP in both spatial and wavelet domains, seven feature categories are made available for dorsal hand vein recognition, with six coming from the wavelet decomposed images (with the non-informative diagonal wavelet component discarded) in addition to that from the original image. For an enrolled image in the database, let $F_{m, o}$ denote its multi-radius LBP feature vector of the partitioned image in either spatial or wavelet domain with $m \in[0,2]$ and $o$ $\in[a, h, v]$. If the corresponding feature vector of the input image to be identified is denoted by $Q_{m, o}$, then the matching score between them based on the Euclidean distance is given by

$$
D\left(F_{m, o}, Q_{m, o}\right)=\sqrt{\sum_{l} \sum_{k}\left[F_{m, o}(l, H)-Q_{m, o}(l, H)\right]^{2}}
$$

Applying equation (8) to each feature category in the spatial and wavelet domains yields seven possible matching scores, and a simple fusion approach to compute the final matching score is to average across scores. However, different feature categories provide different recognition performance, and the category with higher discriminative power should contribute more to the final matching score. This leads to the score weighted fusion given by

$$
D_{c}=\sum_{m} \sum_{o} c_{m, o} D_{m, o}
$$

where $c_{m, o}$ denotes the weighting factors. By using a training data set to learn the recognition performance of each feature category and denoting it as $S_{m, o}$, the weighting factors can be estimated as

$$
c_{m, o}=\frac{S_{m, o}}{\sum_{m} \sum_{o} S_{m, o}}
$$

\section{Results and discussion}

By using the dorsal hand vein image database, this section presents the experiments conducted to yield appropriate weighting factors and to demonstrate the performance of the proposed approach.

\subsection{Wavelet-domain local binary patterns}

The first set of experiments was to obtain appropriate weighting factors for wavelet-domain LBP at one sampling radius by investigating the recognition performance of each feature category as well as score weighted fusion.

With the ROI extracted from each dorsal hand vein image and normalised to $256 \times 256$ pixels, the 2-level Haar wavelet transform was applied to give the approximation, horizontal and vertical wavelet components with a size of $128 \times 128$ pixels at the first decomposition level and $64 \times 64$ pixels at the second decomposition level. By dividing ROI into $8 \times 8$ blocks as well as each of the wavelet components into $4 \times 4$ blocks at the first decomposition level and $2 \times 2$ blocks at the second decomposition level to give a partitioned image size of $32 \times 32$ pixels per block, $L B P_{8,2}^{r i U 2}$

was applied to each block to generate the corresponding LBP histogram, and the resulting histograms are concatenated to form seven feature categories for each dorsal hand vein image, namely one feature vector for ROI denoted by $F_{0}$ with a length of $10 \times 8 \times 8$, three feature vectors for the wavelet components at the first decomposition level denoted by $F_{1, o}$ with each having a length of $10 \times 4 \times 4$ and three feature vectors for the wavelet components at the second decomposition level denoted by $F_{2, o}$ with each having a length of $10 \times 2 \times 2$.

The database of 2040 dorsal hand vein images acquired from 204 hands were divided into two parts. With ten hand vein images per hand, half of them from each hand were randomly selected to form the set of enrolled images and the remaining half was used to form the set of test images. Identification of a test image as belonging to a particular hand in the enrolled set is based on the minimum Euclidean distance computed using equation (8), and performance evaluation is based on the rank-1 recognition rate defined as the percentage of correctly recognised images. 
Table 1 Rank-1 recognition rates of ROI and wavelet-domain one-radius LBP

\begin{tabular}{lccccccc}
\hline Feature vector & $F_{0}$ & $F_{1, a}$ & $F_{1, h}$ & $F_{1, v}$ & $F_{2, a}$ & $F_{2, h}$ & $F_{2, v}$ \\
\hline Rank-1 recognition & $98.33 \%$ & $95.78 \%$ & $92.65 \%$ & $95.10 \%$ & $82.16 \%$ & $44.12 \%$ & $72.06 \%$ \\
\hline
\end{tabular}

From Table 1 showing the recognition results obtained based on seven different feature categories, the relative performance is seen to be consistent with the image information provided by ROI and each wavelet component. From the viewpoint of decomposition levels, the feature vector of a particular orientation at a lower resolution level always yields a lower recognition rate than its counterpart at a higher resolution level, owing to loss of information through down sampling. From the viewpoint of wavelet components, the feature vector of the horizontal component yields a lower recognition rate than those of the vertical component and the approximation component, owing to very few veins running along the horizontal direction. As a result, the feature vector of ROI before wavelet decomposition is seen to provide the highest recognition rate of $98.33 \%$, and the feature vector of the horizontal wavelet component at the second decomposition level is seen to provide the lowest recognition rate of $44.12 \%$. For comparison of relative discriminative power with respect to the original LBP approach, applying $L B P_{8,2}^{r i U 2}$ to ROI without image partition yields a recognition rate of $95.49 \%$, around $3 \%$ lower than that produced by $F_{0}$ with ROI divided into $8 \times 8$ blocks.

Substituting the recognition rates of seven feature categories listed in Table 1 into equation (10) yields the weighting factors shown in Table 2. Applying these weighting factors to yield the Euclidean distance and to find the nearest neighbour in the enrolled set for each of the test image, the rank-1 recognition rate was found to reach $99.02 \%$, which is $0.69 \%$ higher than that without using wavelet-domain LBP.

\subsection{Wavelet-domain multi-radius local binary patterns}

The second set of experiments was to extend score weighted fusion of feature categories based on LBP at one sampling radius in the wavelet domain to LBP at multiple sampling radii by investigating the recognition performance as a function of the number of partitioned image blocks and LBP sampling radius. For the former, the extracted ROI was divided into $8 \times 8,16 \times 16$ and $32 \times 32$ blocks. For the latter, and the sampling radius of $L B P_{8,2}^{r i U 2}$ was increased from 1 to 8 pixels in step of 1 pixel.

Table 2 Weighting factors for ROI and wavelet-domain oneradius LBP

\begin{tabular}{ccccccc}
\hline$c_{0}$ & $c_{1, a}$ & $c_{1, h}$ & $c_{1, v}$ & $c_{2, a}$ & $c_{2, h}$ & $c_{2, v}$ \\
\hline 0.1695 & 0.1651 & 0.1597 & 0.1639 & 0.1416 & 0.0760 & 0.1242 \\
\hline
\end{tabular}

Using the same set-up as the first set of experiments with half of the database used as enrolled images and the other half for testing, Table 3 shows the rank-1 recognition rates obtained by applying the two-level Haar wavelet transform and fusing the resulting LBP-based feature categories based on the weighting factors in Table 2 . With the normalised ROI size of $256 \times 256$ pixels, the block size reduces from $32 \times 32$ pixels to $8 \times 8$ pixels as the number of partitioned image blocks increases from $8 \times 8$ blocks to $32 \times 32$ blocks, and this results in maximum LBP sampling radius of 7 pixels for $16 \times 16$ blocks and 3 pixels for $8 \times 8$ blocks. From Table 3 , there is a trend in the recognition performance which is seen to decrease as the number of partitioned image blocks and the LBP sampling radius increase. Furthermore, the recognition performance for the case of image partition based on $8 \times 8$ blocks is seen to have least variation with the recognition rate staying around $99 \%$.

Table 3 Rank-1 recognition rates as a function of image blocks and sampling radius

\begin{tabular}{cccc}
\hline $\begin{array}{c}\text { LBP sampling } \\
\text { radius (pixels) }\end{array}$ & $\begin{array}{c}8 \times 8 \\
\text { blocks }\end{array}$ & $\begin{array}{c}16 \times 16 \\
\text { blocks }\end{array}$ & $\begin{array}{c}32 \times 32 \\
\text { blocks }\end{array}$ \\
\hline 1 & $98.92 \%$ & $98.73 \%$ & $98.24 \%$ \\
2 & $99.02 \%$ & $98.53 \%$ & $98.04 \%$ \\
3 & $99.02 \%$ & $98.43 \%$ & $96.76 \%$ \\
4 & $98.82 \%$ & $98.53 \%$ & \\
5 & $98.92 \%$ & $98.04 \%$ & \\
6 & $99.02 \%$ & $98.04 \%$ & \\
7 & $98.73 \%$ & $95.69 \%$ & \\
8 & $98.92 \%$ & & \\
\hline
\end{tabular}

From Table 3, the highest recognition rate of $99.02 \%$ is seen to occur at the LBP sampling radii of 2, 3 and 6 pixels with the number of image blocks set to $8 \times 8$. Since different local grey-level variation characteristics are provided by different LBP sampling radii, this leads to further concatenation of the histograms produced by $L B P_{8,2}^{r i U 2}$, $L B P_{8,3}^{r U 2}$ and $L B P_{8,6}^{r i U 2}$ to provide a more comprehensive feature vector for each feature category, so as to increase the recognition performance. As a result, the feature vector length is increased to $10 \times 4 \times 4 \times 3$ for each wavelet component at the first decomposition level, and $10 \times 2 \times 2 \times 3$ for each wavelet component at the second decomposition level. Table 4 shows the rank- 1 recognition results obtained based on the multi-radius LBP feature vectors extracted from six wavelet components.

Table 4 Rank-1 recognition rates of wavelet-domain multiradius $\mathrm{LBP}$

\begin{tabular}{lcccccc}
\hline $\begin{array}{l}\text { Feature } \\
\text { vector }\end{array}$ & $F_{1, a}$ & $F_{1, h}$ & $F_{1, v}$ & $F_{2, a}$ & $F_{2, h}$ & $F_{2, v}$ \\
\hline $\begin{array}{l}\text { Rank-1 } \\
\text { recognition }\end{array}$ & $97.84 \%$ & $94.41 \%$ & $97.35 \%$ & $93.53 \%$ & $90.98 \%$ & $97.25 \%$ \\
\hline
\end{tabular}


Compared with Table 1, the recognition rate shown in Table 4 for each wavelet component is always higher than the corresponding one in Table 1. Furthermore, there is less variation in the recognition performance. A big improvement in recognition performance is seen to occur for the horizontal wavelet component at the second decomposition level with an increase of $47 \%$ as a result of using the multi-radius LBP instead of one-radius LBP. Applying weighted fusion by substituting the recognition rates listed in Table 4 into equation (10), Table 5 shows the weighting factors obtained. Applying these weighting factors to yield the Euclidean distance and to find the nearest neighbour in the enrolled set for each of the test image, the rank-1 recognition rate was found to reach $99.12 \%$.

Table 5 Weighting factors for wavelet based multi-radius LBP

\begin{tabular}{ccccccc}
\hline$c_{0}$ & $c_{1, a}$ & $c_{1, h}$ & $c_{1, v}$ & $c_{2, a}$ & $c_{2, h}$ & $c_{2, v}$ \\
\hline 0.1467 & 0.1461 & 0.1410 & 0.1454 & 0.1397 & 0.1359 & 0.1452 \\
\hline
\end{tabular}

\subsection{Performance evaluation}

With the score weighted fusion of wavelet-domain multiradius LBP found to yield the highest recognition rate, the third set of experiments was performed to compare the recognition performance of the proposed approach with the traditional multi-radius LBP.

With the extracted ROI from each dorsal hand vein image divided into $8 \times 8$ blocks, Figure 5 shows the maximum, average and minimum recognition rates against the number of enrolled images obtained from ten repeated experiments based on different combinations of enrolled and test images. While Figure 5(a) shows the recognition performance of the traditional multi-radius LBP descriptor which was formed by concatenation of the histograms produced by $L B P_{8,2}^{r i U 2}, L B P_{8,3}^{r i U 2}$ and $L B P_{8,6}^{r i U 2}$, as its feature vector, Figure $5(\mathrm{~b})$ shows the recognition performance of the proposed approach based on wavelet-domain multiradius LBP by using the weighting factors listed in Table 5 . Although both approaches have similar trends of increasing recognition rate and decreasing performance variation as the number of training images increase, the proposed approach is seen to offer higher recognition rates with less variation. The average recognition rate of the proposed approach is seen to increase from $91.59 \%$ for one training image to 99.12\% for nine training images as shown in Figure 5(b), which are more than $10 \%$ and $1 \%$ higher than $81.29 \%$ and $97.94 \%$ produced by the traditional multi-radius LBP descriptor as shown in Figure 5(a). Furthermore, the performance variation of the proposed approach is two times less, from $6.54 \%$ (with minimum and maximum recognition rates of $87.25 \%$ and $93.79 \%$ ) for one training image to $3.43 \%$ (with minimum and maximum recognition rates of $96.57 \%$ and $100 \%$ ) for nine training images shown in Figure 5(b), compared with the traditional multi-radius LBP descriptor from $13.07 \%$ (with minimum and maximum recognition rates of $71.13 \%$ and $84.20 \%$ ) to $6.86 \%$ (with minimum and maximum recognition rates of $92.65 \%$ and 99.51\%) shown in Figure 5(a). Based on the rate of change in recognition performance and variation, a recommendation may also be made to use five enrolled images of each hand class.

Figure 5 Average, maximum and minimum of recognition rates. (a) Traditional multi-radius LBP; (b) wavelet-domain multi-radius LBP

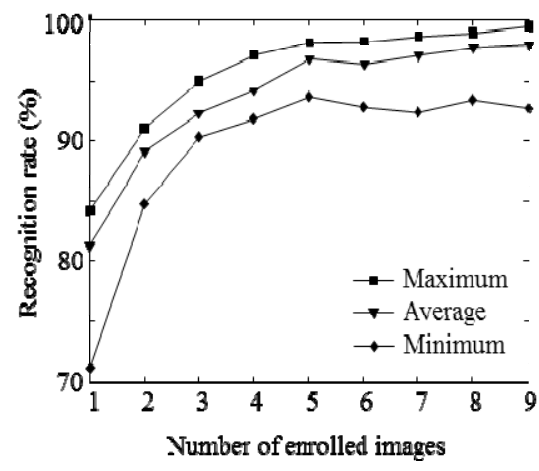

(a)

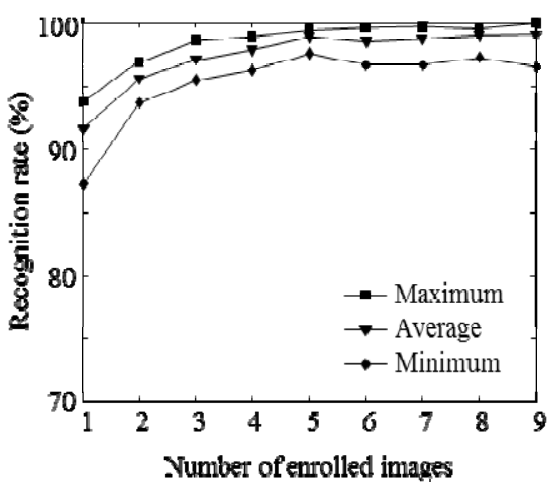

(b)

By dividing the whole database into two halves with five images in each hand class used as enrolled images and the remaining five images used for testing, the curves of FAR and FRR against the recognition threshold, based on the traditional multi-radius LBP and the proposed waveletdomain multi-radius LBP are shown in Figures 6(a) and 7(a), respectively. While FAR was computed based on the Euclidean distances between the feature vectors of each test image of each hand class and all the enrolled images in different hand classes and corresponds to the percentage of the distances above the recognition threshold, FRR was computed based on the Euclidean distance of the feature vector of each test image of each hand class and all other enrolled images of the same hand class and corresponds to the percentage of the distances below the recognition threshold. With the threshold increasing from zero in steps of 0.01 , the proposed approach is seen to have a lower EER of $1.79 \%$ at a lower threshold value of 0.713 as shown in Figure 7(a), compared with the traditional approach with a higher EER of $3.92 \%$ at a higher threshold of 0.746 . For visualisation of the trade-off between FAR and FRR, see Figures 6(b) and 7(b), which show the corresponding receiver operating characteristic curve. 
Figure 6 Traditional multi-radius LBP. (a) FAR and FRR curves; (b) ROC

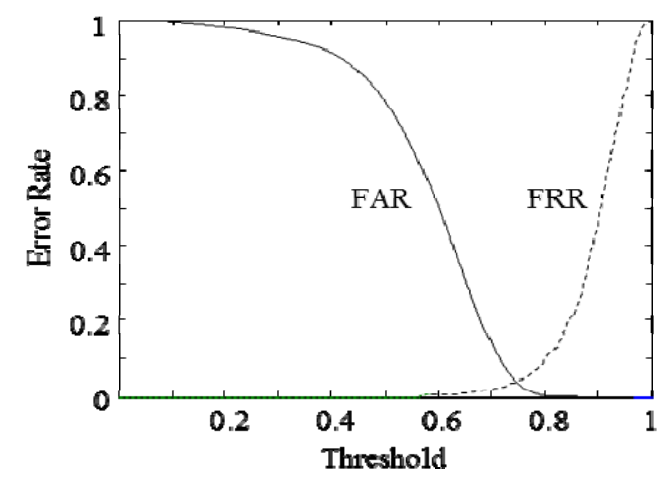

(a)

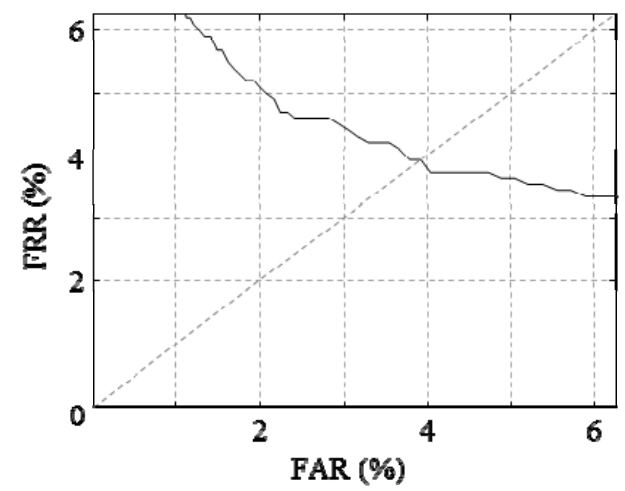

(b)

Figure 7 Wavelet based multi-radius LBP. (a) FAR and FRR curves; (b) ROC

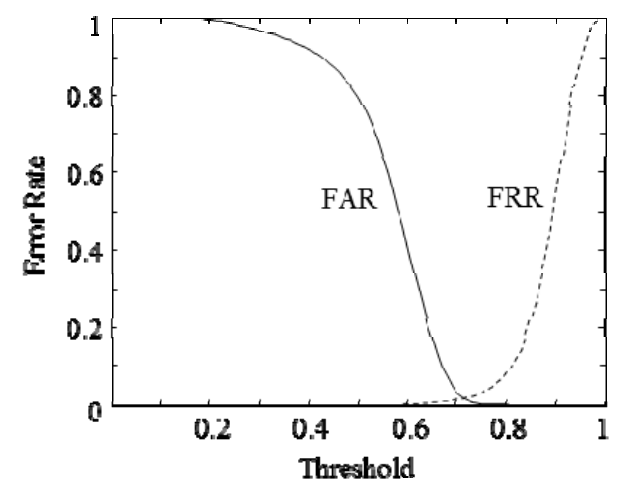

(a)

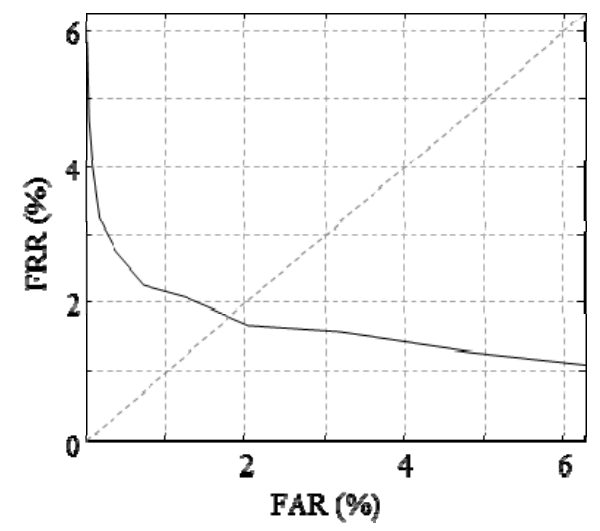

(b)

\section{Conclusions}

This paper presents a method to provide a more comprehensive feature vector of NIR dorsal hand vein images, thereby improving its biometric recognition performance. In the work, the original LBP approach has been extended in three ways. First, it is applied not only in the spatial domain, but also in the wavelet domain to capture grey-level variation characteristics of vein patterns at different orientations and scales. Second, LBP is computed based on partitioned images in both spatial and wavelet domains to capture grey-level variation characteristics of vein patterns at the block level instead of global appearance, and this has the effect of increasing the statistical differences among the LBP histograms derived from different individuals with similar global image characteristics. Third, LBP computation in the wavelet domain is extended from one sampling radius to multiple sampling radii to capture the most discriminative grey-level variation characteristics of vein patterns in a larger area.

The three extensions of the traditional LBP approach were built on investigation of the performance impact made by each component on hand vein recognition. For the wavelet orientation, the diagonal wavelet component was shown to be non-informative as it contains no apparent vein patterns, and the vertical wavelet component was shown to provide a higher recognition rate than that of the horizontal component owing to the dominant vertical orientation of hand vein patterns. For the wavelet resolution, lower decomposition levels were shown to provide a lower recognition performance, and this can be attributed to loss of information as a result of down sampling. For the number of image partitions and sampling radii, increasing their values tend to decrease the recognition performance in general, and the best recognition rate was shown with the number of image partitions set to $8 \times 8$ blocks and the sampling radii set to 2, 3 or 6 pixels.

The improvement in recognition performance achieved by score weighted fusion of wavelet-domain multi-radius LBP can be seen. Using the recognition performance of $95.49 \%$ achieved by the original LBP approach as the baseline, adopting image partition for LBP computation based on $8 \times 8$ blocks is shown to increase the recognition performance to $98.33 \%$, adding wavelet-domain LBP with one sampling radius is shown to raise the recognition performance to $99.02 \%$ and increasing sampling radius from one to three is shown to achieve the highest recognition rate of $99.12 \%$. A comparison with the traditional multi-radius LBP has also been made, and the proposed approach is shown to offer a recognition performance that is more robust with less variation as well as a higher recognition rate and lower EER.

As a direction of future research, the proposed approach could be practically applied to low-quality dorsal hand vein images and to other biometric image modalities to increase recognition rate and to reduce performance variation. With the emerging challenge of heterogeneous images as a result of accommodating multiple devices for biometric acquisition in a distributed and big data environment, other directions for future research include biometric recognition of heterogeneous dorsal 
hand vein images and further development of the proposed approach to address the new biometric challenges.

\section{Acknowledgements}

This work was supported by National Natural Science Foundation of China (Grant No. 61271368), Beijing Natural Science Foundation (Grant No. KZ201410009012 and 4142032) and the Research Program of State Key Laboratory of Software Development Environment (Grant No. SKLSDE2015ZX-30).

\section{References}

Cross, J.M. and Smith, C.L. (1995) 'Thermographic imaging of the subcutaneous vascular network of the back of the hand for biometric identification', Proceedings of the IEEE 29th Annual International Carnahan Conference on Security Technology, 18-20 October, Sanderstead, UK, pp.20-35.

Delac, K. and Grgic, M. (2004) 'A survey of biometric recognition methods', Proceedings of the 46th International Symposium on Electronics in Marine, Zadar, Croatia, 18 June, pp.184-193.

Ding, Y., Zhuang, D. and Wang, K. (2005) 'A study of hand vein recognition method', Proceedings of the IEEE International Conference on Mechatronics \& Automation, 29 July1 August, Niagara Falls, Canada, pp.2106-2110.

Honarpisheh, Z. and Faez, K. (2013) 'An efficient dorsal hand vein recognition based on firefly algorithm', International Journal of Electrical \& Computer Engineering, Vol. 3, No. 1, pp.30-41.

Kumar, A. and Prathyusha, K.V. (2009) 'Personal authentication using hand vein triangulation and knuckle shape', IEEE Transactions on Image Processing, Vol. 18, pp.2127-2136.

Lin, C.L. and Fan, K-C. (2004) 'Biometric verification using thermal images of palm-dorsa vein patterns', IEEE Transactions on Circuits and Systems for Video Technology, Vol. 14, No. 2, pp.199-213.

Mu, M., Ruan, Q. and Song, G. (2011) 'Shift and gray scale invariant features for palmprint identification using complex directional wavelet and local binary pattern', Neurocomputing, Vol. 74, pp.3351-3360.

Ojala, T., Pietikainen, M. and Harwood, D. (1996) 'Comparative study of texture measures with classification based on feature distributions', Pattern Recognition, Vol. 29, No. 1, pp.51-56.

Ojala, T., Pietikainen, M. and Maenpaa, T. (2002) 'Multiresolution grey-scale and rotation invariant texture classification with local binary patterns' IEEE Transactions on Pattern Analysis and Machine Intelligence, Vol. 24, No. 7, pp.971-987.
Otsu, N. (1979) 'A threshold selection method from grey-level histograms', IEEE Transactions on Systems, Man, and Cybernetics, Vol. 9, No. 1, pp.62-66.

Shah, G., Shirke, S., Sawant, S. and Dandawate, Y.H. (2015) 'Palm vein pattern-based biometric recognition system' International Journal of Computer Applications in Technology, Vol. 52, No. 2, pp.105-111.

Song, T. and Li, H. (2013) 'WaveLBP based hierarchical features for image classification', Pattern Recognition Letters, Vol. 34, pp.1323-1328.

Tang, Y., Huang, D. and Wang, Y. (2012) 'Hand-dorsa vein recognition based on multi-level keypoint detection and local feature matching', Proceedings of the 21st International Conference on Pattern Recognition, 11-15 November, Tsukuba, Japan, pp.2837-2840.

Wang, L., Leedham, G., and Cho, S.Y. (2007) 'Infrared imaging of hand vein patterns for biometric purposes', IET Computer Vision, Vol. 1, Nos. 3-4, pp.113-122.

Wang, L., Leedham, G., and Cho, D.S-Y. (2008) 'Minutiae feature analysis for infrared hand vein pattern biometric', Pattern Recognition, Vol. 41, pp.920-929.

Wang, Y. and Zhao, Z. (2013) 'Liveness detection of dorsal hand vein based on the analysis of Fourier spectral', Lecture Notes in Computer Science, Vol. 8232, pp.322-329.

Wang, Y., Li, K., Cui, J., Shark, L-K. and Varley, M.R. (2010) 'Study of hand-dorsa vein recognition', Lecture Notes in Computer Science, Vol. 6215, pp.490-498.

Wang, Y., Li, K., Shark, L-K. and Varley, M.R. (2011) 'Handdorsa vein recognition based on coded and weighted partition local binary patterns', Proceedings of the International Conference on Hand-Based Biometrics, 17-18 November, Hong Kong, pp.1-5.

Wang, Y., Zhang, K. and Shark, L-K. (2014) 'Personal identification based on multiple keypoint sets of dorsal hand vein images', IET Biometrics, Vol. 3, No. 4, pp.234-245.

Wang, Y., Xie, W., Yu, X. and Shark, L-K. (2015) 'An automatic physical access control system based on hand vein biometric identification', IEEE Transactions on Consumer Electronics, Vol. 61, No. 3, pp.320-327.

Yuksel, A., Akarun, L. and Sankur, B. (2011) 'Hand vein biometry based on geometry and appearance methods', IET Computer Vision, Vol. 5, No. 6, pp.398-406.

Zhu, X. and Huang, D. (2012) 'Hand dorsal vein recognition based on hierarchically structured texture and geometry features', Lecture Notes in Computer Science, Vol. 7701, pp.157-164.

Zhu, X., Huang, D. and Wang, Y. (2013) 'Hand dorsal vein recognition based on shape representation of the venous network', Lecture Notes in Computer Science, Vol. 8294, pp.158-169. 